\title{
Wearable Sensors for Assisted Living in Elderly People
}

\author{
Hugo Saner ${ }^{1,2 *}$ \\ ${ }^{1}$ University Clinic of Cardiology, University Hospital Bern, Bern, Switzerland, ${ }^{2} A R T O R G$ Research Center \\ for Biomedical Engineering, University of Bern, Bern, Switzerland
}

Keywords: wearable sensors, ambient sensors, remote monitoring, assisted living, frailty

\section{WEARABLE SENSORS HAVE A GREAT POTENTIAL TO MONITOR HEALTH STATUS OF ELDERLY PEOPLE AND TO CONTRIBUTE TO KEEP INDEPENDENCE}

The number of elderly people is rapidly growing as a proportion of populations in most developed countries around the world. The proportion of the population aged $\geq 65$ years is expected to peak at 25\% of the total population around 2050 (ELSA: English Longitudinal Study of Aging, 2002-2017; HRS: Health and Retirement Study, 2010; SHARE: Survey of Health, Aging and Retirement in Europe, 2006-2017; Chatterji et al., 2015). Most elderly people live at home. However, aging involves decreased mobility, decreased strength and stamina, and reduced sensory acuity. Frailty develops as a consequence of age-related decline in many physiological parameters leading to increased risk of falls, progressive disability, need for long-term care, and increased mortality. Frailty diagnosis

OPEN ACCESS

Edited by:

Norbert Noury,

Université de Lyon, France

Reviewed by:

Martin Cerny,

Technical University

of Ostrava, Czechia

loannis Tarnanas,

ETH Zurich, Switzerland

*Correspondence:

Hugo Saner

hugo.saner@insel.ch

Specialty section:

This article was submitted

to Digital Health,

a section of the journal

Frontiers in ICT

Received: 29 May 2017

Accepted: 12 January 2018

Published: 12 February 2018

Citation:

Saner H (2018) Wearable Sensors for Assisted Living in Elderly People.

Front. ICT 5:1.

doi: 10.3389/fict.2018.00001 is mainly based on grip strength, walking speed (gait speed), clinical frailty scales, self-reported exhaustion, low physical activity, and non-intentional weight loss. All these parameters can be measured by existing devices and may be used to monitor health status and indicators of frailty. Monitoring of these parameters is used to get preventive as well as emergency information. The goal is to maintain a good health status, to prevent frailty, to improve quality of life, to increase safety, and to avoid false alarms.

The advantages of collecting longitudinal data about people's physiological, physical, psychological, cognitive, and behavioral data combined with the recent availability of technologies to enable such data collection, have led to important research activities to develop wearable sensors and sensor networks to be used in the healthcare domain. Major fields of interest are lifestyle interventions for disease prevention, monitoring of chronic diseases to prevent or slow disease progression and assisted living in elderly people. We report about practical aspects and challenges with the integration of wearable devices into an existing ambient sensor system with the aim to maintain a good health status and retard frailty in elderly people living at home.

Traditionally, a person's daily functioning is assessed by means of questionnaires such as the Stanford Health Assessment Questionnaire (Bruce and Fries, 2003) and the Katz Activities of Daily Living Scale (Katz et al., 1963). Due to the fact that these questionnaires rely on self-reporting, they suffer from biases and errors related particularly to cognitive impairment of patients (Carlsson et al., 2012). Subsequently, several research groups have proposed monitoring systems to quantify activity of daily living (ADL) without the disadvantages of self-reporting: they include the use of presence in room (PIR) sensors and wearable sensor systems.

In a recent study on 85 independently living older adults, Petersen et al. (2015) showed that the time spent out of home measured by an activity sensor platform is positively correlated with better cognitive function and with an increased rating on the clinical dementia rating scale (Morris, 1997). The authors find that, while longitudinal measurements are insightful, more fine-grained activity detection would be helpful (Petersen et al., 2015). In another study, Hayes et al. (2008) recorded and 
analyzed more than 108,000 person-hours of continuous activity data based on PIR sensors. They conclude that the transition from healthy to mild cognitive impairment could be detected using relatively basic activity pattern recognition. Research by Merilahti et al. (2015) studied 16 nursing home residents for 12-18 months and found that decreased activity levels and activity regularity were associated with decreased physical functioning and decreased daily stability. They recommend a multivariate monitoring approach to account for interperson variability. Previous work by Stucki et al. (2014) investigated the classification of ADL using an environmental sensor network from within the homes of $10 \mathrm{AD}$ patients and healthy controls over a period of 20 days. We found that it was possible to distinguish AD patients from healthy controls based on eight recognized ADL (Stucki et al., 2014). Finally, in a review by Peetoom et al. (2014), the authors conclude that research focus has remained on small-scale studies so far, and longitudinal studies are necessary to judge the accuracy and usefulness of sensor-based ADL quantification.

An important next step in research will therefore be the long-term quantitative assessment of ADL profile with the aim to determine whether there are inter- and intrapersonal differences (i.e., between persons and over time) in ADL profiles and how the ADL profiles compare to standard neurocognitive tests and measurements of muscular strength and functional mobility. Important clinical results will be an improved understanding how the progression of neurodegenerative diseases or the recovery from stroke influence the ADL profiles of the study participants, how ADL is correlated with functional mobility and with muscular strength, and what is the correlation between functional mobility, muscular strength, cardiovascular function and cognitive decline.

\section{COMBINATION OF DIFFERENT SENSOR DATA IS NECESSARY TO ACQUIRE CLINICALLY USEFUL INFORMATION FOR ADL PROFILES}

Sensor data are best collected combining data from fixed installed ambient motion sensors with wearable devices. The wearable device collects motion/activity data from 3D accelerometers and physiological data. Examples of such devices are the Biovotion monitor (http://www.biovotion.com), the HealthPatch MD, the Preventice BodyGuardian, the Jamar Plus+ dynamometer, the Apple watch or the Fitbit Charge HR or Fitbit-Flex. The sensor data consist of the following variables:

- Actimetry (acceleration of the participant wrist);

- Temperature;

- Light intensity (no visual information);

- Near-field indoor location (only near/far inference possible);

- GPS location when outdoor;

- Physiological measures based on wrist-worn optical and wearable patchsensors, as well as muscle force based on a digital dynamometer; and

- Skin conductivity (also wrist-worn).

\section{SELECTION OF SENSORS HAVE TO BE ADAPTED TO SPECIFIC NEEDS OF ELDERLY PEOPLE}

Procedures to combine a monitoring system using wearable sensors with an existing ambient sensor system include the selection of sensors and platforms, the preparation of informatics/big data analysis, the selection of study subjects according to specific inclusion and exclusion criteria, the predefinition of vital signs interpretation and alerts, and the preparation of the evaluation process including measurements and questionnaires.

Specific requirements for practical use of wearable sensors are the following:

- Different adhesives to ascertain adhesion without skin irritation (sensitive, normal, and strong) if close contact to the body surface is necessary;

- Easy to apply and to change;

- Good battery capacity to avoid frequent battery change/ charging on site;

- Good data quality (signal to noise ratio);

- Reliable data; and

- Adequate platform for data collection.

Potential extensions of a wearable sensor system include data accessibility from the web (tablet and smartphone), web-based communication, e-learning/training, text messaging, artificial intelligence, and additional opportunities for interventions (physical or psychological) (Bhavnani et al., 2016; Rumsfeld et al., 2016).

The selection of the sensor parameters depends on their utility to provide either preventive or emergency information. The most important parameters for monitoring elderly people long term are summarized in Table $\mathbf{1 .}$

Long-term registration of the ECG is regularly used as a standard procedure for the evaluation of heart rhythm and rhythm disturbances, but usually only over $24 \mathrm{~h}$ or a maximum of several days. The possibility of long-time ECG signal acquisition is limited primarily by the adhesive material, by limited battery life time and by the patient's comfort. However, it is evident that

TABLE 1 | Sensor parameters.

\begin{tabular}{ll}
\hline ECG & - Preventive: heart rate and conduction time atrial/ \\
& ventricular stability \\
& - Acute: correlation with sensor signals indicating \\
& emergency \\
- Preventive: decrease over time (frailty, Parkinson, & dementia, and sleep) \\
& - Acute: avoid false alarms and recognize stress \\
Heart rate variability & - Preventive: stress (combined with HR) \\
Respiration rate & - Parkinson, dementia and sleep disorders \\
& - Preventive: lung disease, heart failure, and sleep disorders \\
& fever) \\
\hline Accelerometer (3D) & - Preventive: physical activity and sleep disorders \\
Step counter & - Acute: avoid false alarms (falls) and body posture \\
Skin temperature & - Fever, overheat in summer, and cold exposure in winter \\
Stress and sleep & - Combination of HR, HRV, RR, and accelerometer
\end{tabular}


long-term ECG registration provides important information that can be used for preventive purposes but also in case of emergencies. On the other hand, ECG information can also be used to avoid false alarms. One example is when a motion sensor from a fixed installed ambient infrared motion sensor indicates a fall as an emergency situation, the presence of a stable heart rate with normal ECG allows to exclude a serious event. The major challenges for using ECG tracings over long periods such as months or even years in a specific subject is a reliable analysis and a useful interpretation system that concentrates on those parameters that are useful either for preventive or for emergency situations.

The most important parameters that can be derived from the ECG tracings are heart rate and heart rate variability. Heart rate increases acutely with physical exertion and with stress and decreases very quickly during relaxation and sleep. Furthermore, there are diseases such as dementia or Parkinson disease which are usually accompanied by a slow decrease of the basic heart rate over months and years and where this information can be used for the recognition of early disease stages (Haeberlin et al., 2014; Holmqvist et al., 2015; Otto, 2016). Heart rate variability is a well-known parameter for the recognition of stress but also to evaluate sleep quality (Sassi et al., 2015). Respiration rate can be monitored using specific devices that are attached to the subject's chest but respiration rate is more difficult to evaluate reliably if other locations such as the upper arm are used for sensor placement. Respiration rate increases with stress, fever, and with decompensation of lung disease, but also due to fluid congestion in the lungs in case of heart failure.

Other important parameters for prevention and the recognition of emergencies are motion data from accelerometers and step counters. This information is primarily used to recognize physical activity/inactivity and to diagnose sleep disorders. Furthermore, gait speed correlates well with frailty. To measure gait speed with little interference with the seniors' daily activities poses some challenges. Gait speed can either be measured using a step counter with mobile phone to interpolate the speed, using GPS from mobile phone when seniors go outside to get the speed, combination of signals from infrared sensors on the floor in different locations but with known distances between the sensors, measuring the speed between two rooms using motion sensors or measure the speed with a series of motion sensors on the wall or at the ceiling of a corridor.

In a preventive way, this information is used to recognize and to grade frailty and to detect a decrease of physical activity timely as an indicator of musculoskeletal problems such as joint or back pain, as a sign of declining cardiovascular capacity or in some cases as an indicator of mental problems such as depressive disorders.

\section{CHALLENGES FOR THE INTEGRATION OF BIOSIGNALS INTO AN AMBIENT MOTION SENSOR SYSTEM}

Integration of biosignals from wearable sensors into signals from an existing stationary ambient (motion) sensor system poses important challenges: the additional signals increase the problem of reliable, safe, and secure transmission and analysis of from both systems to achieve maximal benefit for preventive information and reliable emergency recognition. Information from big data has to be transformed into clinically and practically useful information. And visualization of the data in a way that it becomes practically useful for caregivers without adding too much work load is another important challenge. A close cooperation of bioengineers with health informatics specialists and with clinical specialists in the acquisition and interpretation of biosignals is of paramount importance. The most important medical subspecialties to serve this purpose are cardiology, pneumology, and exercise therapy. Another important aspect is to include caregivers such as home care nurses to develop a suitable system which is clinically useful and contributes to achieve our primary goal: to help to allow elderly people to keep independence and to stay at home healthy and with good quality of live as long as possible.

\section{DISCUSSION}

One of the biggest challenges for our societies in the near future is to help the fast growing population of older citizen to live independently in good health and with good quality of life. The combined use of wearable sensors with ambient motion sensors has an enormous potential to achieve this goal.

However, today there is a gap between technological developments and daily clinical use of the technology. One explanation for this gap is the multidisciplinary nature of these support systems. Research in this area has to be planned and performed in close cooperation of bioengineers with medical doctors and care givers. Complementary knowledge is essential for successful translation of research results and their technological development into clinical practice and care. Furthermore, researchers and developers have to evaluate their sensor systems in real world in close cooperation with senior citizens. A major challenge in this regard is the correlation of motion and biological signals with events such as dizziness, falls, cardiovascular events, and with progressive decline of physical and mental performance. Another challenge is to transform big sensor data into a simple, reliable, and practically useful alert and diagnostic system. There are three major areas of interest: to collect preventive information, to recognize emergencies, and to avoid false alarms (for which the use of complementary information from different sensor systems is of particular importance). Our approach may lead to a changing paradigm in regard to the main application of wearable sensors from the use in physical activity tracking and in patients with disease to the use in seniors for preventive information to keep them independent and with good quality of life as long as possible.

\section{AUTHOR CONTRIBUTIONS}

HS drafted the manuscript and was involved in its conception and critical review. 


\section{REFERENCES}

Bhavnani, S. P., Narula, J., and Sengupta, P. P. (2016). Mobile technology and the digitization of healthcare. Eur. Heart J. 37, 1428-1438. doi:10.1093/eurheartj/ ehv770:1-12

Bruce, B., and Fries, J. F. (2003). The Stanford Health Assessment Questionnaire: a review of its history, issues, progress, and documentation. J. Rheumatol. 30, 167-178.

Carlsson, G., Haak, M., Nygren, C., and Iwarsson, S. (2012). Self-reported versus professionally assessed functional limitations in community-dwelling very old individuals. Int. J. Rehabil. Res. 35, 299-304. doi:10.1097/MRR. 0b013e3283544d07

Chatterji, S., Byles, J., Cutler, D., Seeman, T., and Verdes, E. (2015). Health, functioning, and disability in older adults - present status and future implications. Lancet 385, 563-575. doi:10.1016/S0140-6736(14)61462-8

ELSA: English Longitudinal Study of Aging. (2002-2017). Available at: http://www. elsa-project.ac.uk

Haeberlin, A., Roten, L., Schilling, M., Scarcia, F., Niderhauser, T. H., Vogel, R., et al. (2014). Software-based detection of atrial fibrillation in long-term ECGs. Heart Rhythm 11, 933-938. doi:10.1016/j.hrthm.2014.03.014

Hayes, T. L., Abendroth, F., Adami, A., Pavel, M., Zitzelberger, T. A., and Kaye, J. A. (2008). Unobtrusive assessment of activity patterns associated with mild cognitive impairment. Alzheimers Dement. 4, 395-405. doi:10.1016/j.jalz. 2008.07.004

Holmqvist, F., Kim, S., Steinberg, B. A., Reiffel, J. A., Mahaffey, W., Gersh, B. J., et al. (2015). Heart rate is associated with progression of atrial fibrillation, independent of rhythm. Heart 101, 894-899. doi:10.1136/heartjnl-2014307043

HRS: Health and Retirement Study. (2010). Available at: http://hrsonline.isr.umich. edu/modules/meta/2010/core/desc/h10dd.pdf

Katz, S., Ford, A. B., Moskowitz, R. W., Jackson, B. A., and Jaffe, M. W. (1963). Studies of illness in the aged: the index of ADL: a standardized measure of biological and psychosocial function. JAMA 185, 914-919. doi:10.1001/ jama.1963.03060120024016

Merilahti, J., Petteri, V., and Ilkka, K. (2015). Wearable monitoring of physical functioning and disability changes, circadian rhythms and sleep patterns in nursing home residents. IEEE J. Biomed. Health Infom. 20, 856-864. doi:10.1109/ JBHI.2015.2420680
Morris, J. C. (1997). Clinical dementia rating: a reliable and valid diagnostic and staging measure for dementia of the Alzheimer type. Int. Psychogeriatr. 9, 173-6; discussion 177-8. doi:10.1017/S1041610297004870

Otto, C. M. (2016). Heartbeat: detection of arrhythmias in older adults with falls and syncope. Heart 102, 651-652. doi:10.1136/heartjnl-2016-309648

Peetoom, K. K., Lexis, M. A., Joore, M., Dirksen, C. D., and De Witte, L. P. (2014). Literature review on monitoring technologies and their outcomes in independently living elderly people. Disabil. Rehabil. Assist. Technol. 10, 271-294. doi:10.3109/17483107.2014.961179

Petersen, J., Austin, D., Mattek, N., and Kaye, J. (2015). Time out-of-home and cognitive, physical, and emotional wellbeing of older adults: a longitudinal mixed effects model. PLoS ONE 10:e0139643. doi:10.1371/journal.pone. 0139643

Rumsfeld, J. S., Brooks, S. C., Aufderheide, T. P., Leary, M., Bradley, S. M., NkondePrice, C., et al. (2016). Use of mobile devices, social media, and crowd sourcing as digital strategies to improve emergency cardiovascular care. Circulation 134, e87-e108. doi:10.1161/CIR.0000000000000428

Sassi, R., Cerutti, S., Lombardi, F., Malik, M., Huikuri, H. V., Peng, C. H.-K., et al. (2015). Advances in heart rate variability signal analysis: joint position statement by the e-cardiology ESC working group and the European Heart Rhythm association co-endorsed by the Asia Pacific Heart Rhythm Society. Europace 17, 1341-1353. doi:10.1093/europace/euv015

SHARE: Survey of Health, Aging and Retirement in Europe. (2006-2017). Available at: http://www.share-project.org

Stucki, R. A., Urwyler, P., Rampa, L., Müri, R., Mosimann, U. P., and Nef, T. (2014). A web-based non-intrusive ambient system to measure and classify activities of daily living. J. Med. Internet Res. 16, e175. doi:10.2196/jmir.3465

Conflict of Interest Statement: The author declares that the research was conducted in the absence of any commercial or financial relationships that could be construed as a potential conflict of interest.

Copyright (c) 2018 Saner. This is an open-access article distributed under the terms of the Creative Commons Attribution License (CC BY). The use, distribution or reproduction in other forums is permitted, provided the original author(s) and the copyright owner are credited and that the original publication in this journal is cited, in accordance with accepted academic practice. No use, distribution or reproduction is permitted which does not comply with these terms. 